University of Wollongong

Research Online

Faculty of Engineering and Information

Faculty of Engineering and Information

Sciences - Papers: Part A

Sciences

$1-1-2012$

Strain-induced phase transformation during thermo-mechanical processing of titanium alloys

A Dehghan-Manshadi

University of Wollongong, alidm@uow.edu.au

Rian J. Dippenaar

University of Wollongong, rian@uow.edu.au

Follow this and additional works at: https://ro.uow.edu.au/eispapers

Part of the Engineering Commons, and the Science and Technology Studies Commons

Research Online is the open access institutional repository for the University of Wollongong. For further information contact the UOW Library: research-pubs@uow.edu.au 


\title{
Strain-induced phase transformation during thermo-mechanical processing of titanium alloys
}

\author{
Abstract \\ Strain-induced phase transformation studies have been conducted in a Ti-6Al-2Sn-4Zr-6Mo alloy. This \\ alloy was subjected to $b$ sub-transus deformation upon slow cooling from the b-phase field and the effect \\ of strain on the extent and morphology of the phase transformation during hot-deformation was \\ determined. In addition, the transformation kinetics and the morphology of the newly formed a-phase \\ were studied following deformation under different cooling conditions. By applying strain, the rate of the \\ b-to-a phase transformation increased significantly during deformation as well as during slow cooling \\ following deformation. This increase in the kinetics of the b-to-a transformation can be ascribed to \\ straininduced phase transformation. Also, the extent of deformation of the b-phase had a marked effect \\ on the resulting morphology and size of the newly formed a-phase. Transformation of un-deformed b- \\ phase rendered a-phase of acicular morphology only. However, following deformation of the b-phase, \\ acicular as well as globular a-phase morphologies have been observed upon cooling.
}

\section{Keywords}

transformation, phase, induced, titanium, processing, mechanical, thermo, alloys, during, strain

\section{Disciplines}

Engineering | Science and Technology Studies

\section{Publication Details}

Dehghan-Manshadi, A. \& Dippenaar, R. J. (2012). Strain-induced phase transformation during thermomechanical processing of titanium alloys. Materials Science and Engineering A: Structural Materials: Properties, Microstructure and Processing, 552 451-456. 


\title{
Strain-induced phase transformation during thermo- mechanical processing of titanium alloys
}

\author{
Ali Dehghan-Manshadi ${ }^{1, a}$ and Rian J. Dippenaar ${ }^{1}$ \\ 1) Faculty of Engineering, University of Wollongong, Wollongong, NSW 2500, Australia \\ a)Corresponding author, Email: alidm@uow.edu.au, Phone: +61 24221 4859, Fax: +61 242213662
}

Keywords: Thermo-mechanical processing, Strain-induced phase transformation, Titanium alloys, Grain refinement

\begin{abstract}
Strain-induced phase transformation studies have been conducted in a Ti-6Al-2Sn-4Zr-6Mo alloy. This alloy was subjected to $\beta$ sub-transus deformation upon slow cooling from the $\beta$ phase field and the effect of strain on the extent and morphology of the phase transformation during hot-deformation was determined. In addition, the transformation kinetics and the morphology of the newly formed $\alpha$-phase were studied following deformation under different cooling conditions. By applying strain, the rate of the $\beta$-to- $\alpha$ phase transformation increased significantly during deformation as well as during slow cooling following deformation. This increase in the kinetics of the $\beta$-to- $\alpha$ transformation can be ascribed to strain-induced phase transformation. Also, the extent of deformation of the $\beta$-phase had a marked effect on the resulting morphology and size of the newly-formed $\alpha$-phase. Transformation of un-deformed $\beta$-phase rendered $\alpha$-phase of acicular morphology only. However, following deformation of the $\beta$-phase, acicular as well as globular $\alpha$-phase morphologies have been observed upon cooling.
\end{abstract}




\section{Introduction}

A necessary pre-requisite to achieving the desired combination of strength and toughness in metallic materials for advanced applications, is control over microstructure and more specifically the ability to refine grains to micron or sub-micron sizes. It is difficult to attain such ultra-fine grain structures in titanium alloys because of the very rapid rate of grain growth experienced in these alloys, especially at high temperature. However, severe plastic deformation (SPD) techniques such as equal channel angular pressing or high pressure torsion have been utilized to devise sub-micron or even nano-scale structures, albeit only on laboratory scale [1-2]. The major problems encountered with these processes are the limitations placed on size, dimensions and geometry of the components to be manufactured. These limitations have restricted the industrial application of SPD techniques, especially to the production of aerospace parts, which generally are of complex geometries and intricate designs. An alternative approach to producing ultra-fine grain structures in titanium alloys might be to exploit the phase transformation characteristics of these alloys in combination with innovative thermo-mechanical processing techniques.

Ultra-fine microstructures with exceptional combinations of strength and toughness have been produced in a variety of steels through a judicious selection of thermo-mechanical processing techniques and the manipulation of the pertaining phase transformations. This approach, commonly referred to as strain induced phase transformations (SIT), has shown great potential in the development of ultra-fine grain structures not only in steels, but also in other metallic materials [3-4]. Because there are so many parallels between the $\gamma$-to- $\alpha$ phase transformation in iron-based alloys and the $\beta$-to- $\alpha$ phase transformation in titanium-based alloys, there is reason to believe that strain-induced phase transformations in titanium alloys can in similar vein, lead to the development of fine-grained microstructures.

Apart from earlier studies of martensite-based strain-induced transformation following cold deformation [5-7], the merit of manipulating the mechanical properties of candidate titanium alloys by strain-induced phase transformations at high temperature, utilizing the $\beta \leftrightarrow \alpha$ phase transformation, does not seem to have been fully assessed nor exploited, probably due to the complex phase transformations in titanium alloys and the practical difficulties of applying thermo-mechanical processing for the production of components of industrial importance. 
Ti-6Al-2Sn-4Zr-6Mo (Ti-6246) is a heat treatable $\alpha / \beta$ titanium alloy providing very high strength at elevated temperatures and is currently being used in engineering applications, for example gas turbine engine compressors [8-9]. The Ti-6246 alloy is often produced by the so-called hot-die and isothermal-forging process, an example of a cost effective near netshape forming process, specifically for applications in the aerospace industry. It is therefore likely that the excellent mechanical properties produced by this manufacturing technique are a result of hot-deformation and subsequent or concurrent phase transformations that occur in the course of the high temperature processing steps. Also, further phase transformations are likely to occur during cooling of the deformed workpiece, which can further affect the mechanical properties and microstructure of the final product.

The main objectives of the present study were firstly to examine the evolution of microstructure in a commercially produced Ti-6246 alloy as a result of strain-induced phase transformations, following deformation at sub- $\beta$-transus temperatures. Secondly, to study the effect of such hot-deformation on the subsequent phase transformations and micro-structural morphologies that develops during slow cooling.

\section{Experiments}

Rectangular, $17 \times 11 \times 7 \mathrm{~mm}$ samples, complying with the dimensional requirements for planestrain compression tests, were cut from an as-received, commercially available Ti-6246 alloy with chemical composition shown in Table 1. Hot-compression tests were conducted in a Gleeble 3500 thermo-mechanical simulator under vacuum. Samples were heated to $1348 \mathrm{~K}$ at a rate of $10 \mathrm{Ks}^{-1}$ and held for $300 \mathrm{~s}$, aiming to achieve a stable, single-phase $\beta$-microstructure. Samples were then cooled to $1073 \mathrm{~K}$ at a cooling rate of $5 \mathrm{Ks}^{-1}$, followed by deformation to different strains at different strain rates. Following deformation, some samples were directly quenched in water to study the micro-structural development upon hot-deformation and dynamic strain induced phase transformation. Following hot-deformation, other samples were cooled at a rate of $5 \mathrm{Ks}^{-1}$ to different temperatures followed by water quenching in order to assess the influence of hot-deformation on the occurrence of subsequent phase transformations during slow cooling. Figure 1 shows the applied hot deformation routes overlaid on a schematic time-temperature -transformation (TTT) diagram of the Ti-6246 alloy. Microstructural development was studied by optical and scanning electron microscopy (SEM) following standard metallographical practice. Figure 1 shows a schematic of the 
Gleeble samples that were designed in such a way that the deformed and un-deformed areas, that had undergone the exact heat treatment, could be studied in a single sample.

\section{Results}

In order to fully evaluate the influence of deformation on the phase transformations occurring in the selected Ti-6246 alloy, it is pertinent to have a detailed knowledge of the microstructure that exists immediately before deformation. This is induced because the preexisting grain boundaries provide the key nucleation sites for the transformation of the new phase. The samples were annealed at $1348 \mathrm{~K}$, well above the $\beta$-transus temperature $\left(\mathrm{T}_{\beta}=\right.$ $1223 \mathrm{~K}[10])$, and at this temperature, the microstructure consisted of a single $\beta$-phase with a grain size of $500 \mu \mathrm{m}$. It is assumed that the same $\beta$-microstructure prevailed on cooling until deformation was initiated at $1073 \mathrm{~K}$ because no phase transformations are likely to occur between the annealing and deformation temperatures at the applied cooling rate of $5 \mathrm{Ks}^{-1}$. Deformation followed by cooling of this single-phase $\beta$-structure resulted in phase transformations, which could have a significant influence on the resulting microstructure and mechanical properties and hence, these aspects were further explored.

Figure 2 shows a typical true stress-true strain curve as well as the work-hardening rate for the Ti-6246-alloy following prior deformation at a sub- $\beta$-transus temperature of $1073 \mathrm{~K}$ and a strain rate of $1 \mathrm{~s}^{-1}$. The mathematical technique introduced by Kocks and Mecking [11] has been used to calculate the work hardening rate. By this method, work hardening rates, $\theta=\partial \sigma / \partial \varepsilon$, are plotted as a function of stress and work softening phenomena (recovery and recrystallization) are identified by an inflection in the work hardening curve. The flow curve of the selected titanium alloy shown in Figure 2 is characterized by rapid work hardening up to the peak stress, followed by work softening at a lower rate. However, a steady state is never obtained in the flow curve. Figure 2 shows that the initial work hardening rate of about $10,000 \mathrm{MPa}$ during the early stages of deformation, diminishes to zero at peak stress. Beyond the peak stress, there is an inflection in the strain-rate curve, indicating that softening begins to occur at a low rate, which could be attributed to the operation of restoration processes, either dynamic recrystallization (DRX) and/or dynamic recovery [12]. However, there is also the possibility that this work-softening could in part be attributed to the occurrence of the $\beta$ to $-\alpha$ phase transformation. In order to explore these different processes in more detail and in an attempt to identify the rate determining softening mechanism, the deformed as well as the 
un-deformed areas of the Gleeble specimens, which were quenched following deformation to different strains, were examined metallographically.

The metallographic investigation revealed that hot-deformation in the $\beta$-phase field accelerates the transformation of the $\beta$-phase to the $\alpha$-phase, presumably due to an increase in the number of nucleation sites for the nucleation of the $\alpha$-phase. The phase transformation of $\beta$ to $\alpha$ resulted in the formation of two different morphologies of $\alpha$-phase: an equiaxed structure of $\alpha$ grains (globular $\alpha$ ), which mostly formed on the initial $\beta$-grain boundaries and lathes of $\alpha$ (acicular $\alpha$ ) which formed on both $\beta$-grain boundaries and within the $\beta$-grain interiors. Deformation increased the volume fraction of globular $\alpha$ grains, which formed along the initial $\beta$ grain boundaries, much more that of the acicular $\alpha$. In fact no globular $\alpha$ formed in the un-deformed sample, which was subjected to the same heat treatment. Figure 3 shows in a quantitative fashion that the volume fraction (determined by point counting techniques [13]) of the globular $\alpha$-phase gradually increases from zero in un-deformed sample to about $10 \%$ in a sample deformed to a strain of 1.0 . This observation indicates that under the present deformation condition, the kinetics of the $\beta$-to- $\alpha$ (globular) phase transformation is noticeably increased due to dynamic strain-induced transformation (DSIT). However, the fraction of globular $\alpha$-phase, which resulted from the chosen deformation conditions, is not high enough to provide a truly duplex $\alpha+\beta$ grain microstructure. It follows from Figure 3 that a critical minimum strain of about 0.5 is required to initiate the $\beta$-toglobular- $\alpha$ phase transformation. Under the pertaining thermo-mechanical processing conditions, this high critical strain to initiate transformation could be attributed to the very large initial $\beta$-grain size. By increasing the strain beyond the critical point, the serration of initial $\beta$-grain boundaries increases, thereby provides increased opportunities for the nucleation of the $\alpha$-phase. Figure 3 shows that there is an almost linear increase in the volume fraction of $\alpha$-phase as a function of strain. However, at very high strains intragranular sites might be activated for the nucleation of the globular $\alpha$-phase, typically by the formation of deformation bands and the accumulation of dislocations in the grain interiors. These additionally available nucleation sites should increase the fraction of globular $\alpha$-phase formed beyond a linear relationship with strain. 
The influence of deformation on the formation of acicular- $\alpha$, which formed inside the initial $\beta$ grains, was also assessed. High resolution SEM images, such as those superimposed on the optical micrographs in Figure 3, show that the fraction of acicular- $\alpha$ in deformed samples is much higher than that in the un-deformed sample. In fact, in un-deformed sample $\alpha$-plates were formed on the $\beta$-grain boundaries only, but after deformation $\alpha$-plates started to form within the interiors of $\beta$-grains. This noteworthy influence of deformation on the transformation of the $\beta$-phase into $\alpha$-plates could be attributed to the creation of nucleation sites by microstructural defects such as deformation bands and dislocation tangles, within the $\beta$-grains during deformation. The SEM images in Figure 3 reveal that while a large fraction of $\alpha$-plates formed on the grain boundaries as well as inside heavily deformed $\beta$-grains, there is only a small fraction of $\alpha$-plates present on $\beta$-grain boundaries in the less-deformed material. $\alpha$-plates were not observed in un-deformed samples.

It is important to note that a large fraction of the defects created by hot-deformation such as serrated grain boundaries, dislocations and deformation bands remain in the microstructure after deformation and even after the formation of the $\alpha$-phase. Therefore, these defects can further act as sites for the nucleation of the $\alpha$-phase during annealing or slow cooling of deformed specimens. In addition, such defects, which increase the internal energy of the deformed structure, can encourage the growth of previously transformed $\alpha$ during slow cooling. It therefore becomes clear that thermo-mechanical processing can increase the phase transformation kinetics by increasing the number of nucleation sites and also by enhancing the growth of the already formed $\alpha$-phase. In order to understand the behavior of a deformed structure and accelerating the phase transformation during slow cooling, Gleeble samples were slowly cooled subsequent to deformation to a strain of 0.7 at a temperature and strain rate of $1073 \mathrm{~K}$ and $1 \mathrm{~s}^{-1}$ respectively, and some interrupted quench tests were performed by quenching from different temperatures. Following this quench-arrest procedure, the microstructures of the deformed areas of Gleeble samples were analyzed and the volume fraction of globular $\alpha$-phase measured using standard point counting techniques [13]. These microstructures were then compared to the un-deformed areas of Gleeble samples as shown in Figure 4. This figure clearly shows that hot-deformation increases the volume fraction of the globular $\alpha$-phase formed as well as enhancing the kinetics of the $\beta$-to- $\alpha$ phase transformation (specimens were all cooled at a rate of $5 \mathrm{Ks}^{-1}$ ). Only a small number of $\alpha$ grains formed on pre-existing $\beta$-grain boundaries in the un-deformed samples and the volume 
fraction of this globular phase was less than 5\%, even after slow cooling to $673 \mathrm{~K}$. By contrast, after deforming samples to a strain of 0.7 at $1073 \mathrm{~K}$, a volume fraction of the globular $\alpha$-phase of more than $15 \%$ was attained under similar cooling conditions. Microstructural examination, using SEM techniques revealed a similar influence of hotdeformation on the volume fraction of acicular- $\alpha$ formed as has been shown in Figure 4 for the globular $\alpha$-phase.

A more detail examination revealed that a thin (up to $0.3 \mu \mathrm{m}$ ) and continuous layer of $\alpha$ phase was frequently observed on $\beta$-grain boundaries in un-deformed samples. However, this thin layer was discontinuous in deformed samples as shown in Figure 5. Interestingly enough, this fine $\alpha$-precipitates were not only found on un-recrystallized $\beta$-grain boundaries, but also on some $\beta$-grain boundaries where recrystallization has already occurred. Figure $5 \mathrm{~b}$ is an enlargement of the area shown in Figure 5a and shows that recrystallization has already occurred on the deformed $\beta$-grain boundary. As shown in Figure $5 \mathrm{c}$, these $\beta$-grains clearly delineate the newly formed $\beta$-grain boundaries. Those newly formed (recrystallized) $\beta$-grain boundaries were covered by a discontinuous layer of $\alpha$ phase. These $\alpha$-layers can grow during further slow cooling, but it is more likely that new $\alpha$-plates will nucleate on the existing $\alpha$-layers and that they will grow towards the $\beta$-grain interior. Our observations show a much greater extent of this growth process in the deformed areas of samples compared to the un-deformed areas, evidently because of the high dislocation density. It is pertinent to mention that the formation of continuous $\alpha$-layers along $\beta$-grain boundaries is a common occurrence in the microstructural development of $\alpha+\beta$ and near $\beta$-alloys, resulting in reduced ductility and fatigue performance [14]. However, this study has shown that deformation of the $\beta$-phase before the start of the phase transformation caused a discontinuity in the $\alpha$-layers which formed on the $\beta$-grain boundaries as shown in Figure 5c. These discontinuous $\alpha$-layers are expected to be less harmful to the mechanical properties than continuous layers.

\section{Discussion}

Most of the work to date relating to strain-induced phase transformation of titanium alloys, have been concerned with the formation of martensite during cold deformation of different $\mathrm{Ti}$ alloys, specifically near- $\beta$ alloys as a means of developing ultra-fine grained microstructures 
[5, 15-17]. However, the most industrially important titanium components are manufactured from duplex $\alpha+\beta$ alloys, which are usually manufactured by employing a variety of thermomechanical processing techniques above and below the $\beta$-transus temperature, $\mathrm{T}_{\beta}$. It is therefore most likely that a combination of hot-deformation and phase transformation (i.e. strain-induced phase transformation) is already being used in the manufacturing of titanium components.

The present investigation has unmistakably shown that dynamic as well as static straininduced phase transformation of $\beta$-to- $\alpha$ can be effected in a Ti-6246 alloy during and following hot-deformation, respectively. These strain-induced transformation techniques increased the kinetics of the phase transformation, reduced the size of the precipitating $\alpha$ phase and consequently, improved the mechanical properties of this alloy. It was also found that two different morphologies of $\alpha$-phase can develop during thermo-mechanical processing: globular $\alpha$ with an equiaxed grain structure and acicular $\alpha$ with a layered (platelike) structure. The equiaxed $\alpha$ formed mostly, but not exclusively on pre-existing $\beta$-grain boundaries and it is expected that the formation of this phase will improve the toughness of material. The equiaxed $\alpha$ was observed in deformed samples only, but the secondary $\alpha$-phase was observed on both deformed and un-deformed specimens. A schematic illustration of $\alpha$ phase development during thermo-mechanical processing of the selected Ti-6246 alloy is shown in Figure 6 for both the deformed and un-deformed cases. The initial microstructure immediately prior to deformation consists of large and equiaxed grains of the $\beta$-phase. Deformation causes an elongation of the $\beta$-grains and serration of their boundaries. However, the formation of defects such as deformation bands is expected in the grain interiors. Transformation initiates by nucleation of $\alpha$-grains and plates along these serrated $\beta$-grain boundaries. The volume fraction of both the acicular and globular $\alpha$ morphologies increases in the course of deformation. Hence, on completion of the deformation process, the microstructure of a deformed specimen includes elongated $\beta$-grains as well as some small $\alpha$-grains, which nucleated on the $\beta$-grain boundaries alongside some acicular $\alpha$, (Fig 6a). These $\alpha$-grains starts to growth in the course of slow cooling. A large number of small equiaxed $\alpha$-grains forms immediately upon cooling, but during cooling and during the progress of the phase transformation, large grains grow at the expense of the small $\alpha$-grains, (Figures $6 \mathrm{~b}-\mathrm{d}$ ). Small plates of acicular $\alpha$ start to form firstly from the $\beta$-grain boundaries and then within the $\beta$-grains interior during cooling of deformed specimens. On conclusion of 
thermo-mechanical processing, the microstructure of deformed material consists of a small fraction ( $15 \%$ in this instance) of equiaxed $\alpha$-grains with an average size of $60 \mu \mathrm{m}$ and a large number of acicular $\alpha$-plates in the $\beta$-matrix and on $\beta$-grain boundaries as shown in Figure $6 \mathrm{~d}$.

The microstructure of un-deformed specimens can be clearly distinguished from their deformed counterparts. A small number of acicular $\alpha$-plates start to form on $\beta$-grain boundaries in the early stages of cooling and their number increases as cooling proceeds. Also, at the later stages of cooling some acicular $\alpha$-plates form within the $\beta$-grain as shown in Figures 6(f-h). The final microstructure in un-deformed specimens contains acicular $\alpha$-plates within the $\beta$-matrix and on $\beta$-grain boundaries (Figure $6 \mathrm{~h}$ ).

\section{Conclusions}

The concurrent phase transformation and hot-deformation of a Ti-6Al-2Sn-4Zr-6Mo alloy, studied by hot-deformation of rectangular samples in a Gleeble thermo-mechanical simulator, revealed that strain-induced phase transformation techniques can be usefully employed to manipulate the microstructural development.

Deformation at temperatures below the $\beta$-transus plays a significant role in the $\beta$-to- $\alpha$ phase transformation kinetics as well as the morphology of the resulting phases. Slow cooling of deformed material displays significantly higher transformation kinetics than an un-deformed structure. A critical minimum strain is required to initiate the $\beta$-to- $\alpha$ phase transformation. Two different morphologies of equiaxed grains, which mostly formed on $\beta$-grain boundaries, and acicular $\alpha$, which formed as small plates on grain boundaries as well as grain interiors, were observed on deformed and slowly cooled samples.

\section{References}

[1] I. Sabirov, M.T. Perez-Prado, J.M. Molina-Aldareguia, I.P. Semenova, G.K. Salimgareeva, R.Z. Valiev, Scripta Mater., 64 (2011) 69-72..

[2] M.R. Shankar, B.C. Rao, S. Lee, S. Chandrasekar, A.H. King, W.D. Compton, Acta Mater., 54 (2006) 3691-3700..

[3] S.J. Li, T.C. Cui, Y.L. Li, Y.L.H. Yang, Applied Phys. Letter, 92 (2008) 04312810431283..

[4] C.J. Huang, H. Zhang, Y.J. Nan, D.Z. Li, Y.Y. Li, Journal of Materials Processing Technology, 160 (2005) 123-127..

[5] Y. Yang, G.P. Li, G.M. Cheng, H. Wang, M. Zhang, F. Xu, K. Yang, Scripta Mater., $58(2008)$ 9-12..

[6] M.-H. Cai, C.-Y. Lee, S. Kang, Y.-K. Lee, scripta Mater., 64 (2011) 1098-1101.. 
[7] S. Nag, R. Banerjee, R. Srinivasan, J.Y. Hwang, M. Harper, H.L. Fraser, Acta Mater., 57 (2009) 2136-2147..

[8] G. Welsch, R. Boyer, E.W. Collings, Materials properties handbook: titanium alloys ASM International, 1994..

[9] M. Jackson, R.J. Dashwood, L. Christodoulou, H.M. Flower, Journal of Light Metals, 2 (2002) 185-195..

[10] P. Tarín, I. Alonso, A.G. Simón, J.M. Badía, N.M. Piris, Mater. Sci. Eng. A, 481-482 (2008) 559-561..

[11] H. Mecking, U.F. Kocks, Acta Metall., 29 (1981) 1865-1875..

[12] A. Dehghan-Manshadi, M.R. Barnett, P.D. Hodgson, Mater. Sci. Eng. A., 485A (2008) 664-672..

[13] R.L. Higginson, C.M. Sellars, Worked Examples in Quantitative Metallography, Maney, 2003..

[14] C. Sauer, G. Lutjering, Mater. Sci. Eng. A., 319-321 (2001) 393-397..

[15] H.Y. Kim, Y. Ikehara, J.I. Kim, H. Hosoda, S. Miyazaki, Acta Mater., 54 (2006) 2419-2429..

[16] F. Zimmermann, M. Humbert, Acta Mater., 50 (2002) 1735-1740..

[17] W. Xu, K.B. Kim, J. Das, M. Calin, B. Rellingghaus, J. Eckert, Applied Phys. Letter, 89 (2006) 031906. 
Table 1 Chemical composition of Ti-6246 alloy

\begin{tabular}{|c|c|c|c|c|c|c|c|c|}
\hline & Al & Sn & Zr & Mo & C & Fe & O & Ti \\
\hline $\mathrm{wt} \%$ & 6.2 & 2.1 & 4.2 & 6.0 & 0.04 & 0.15 & 0.11 & Balance \\
\hline
\end{tabular}




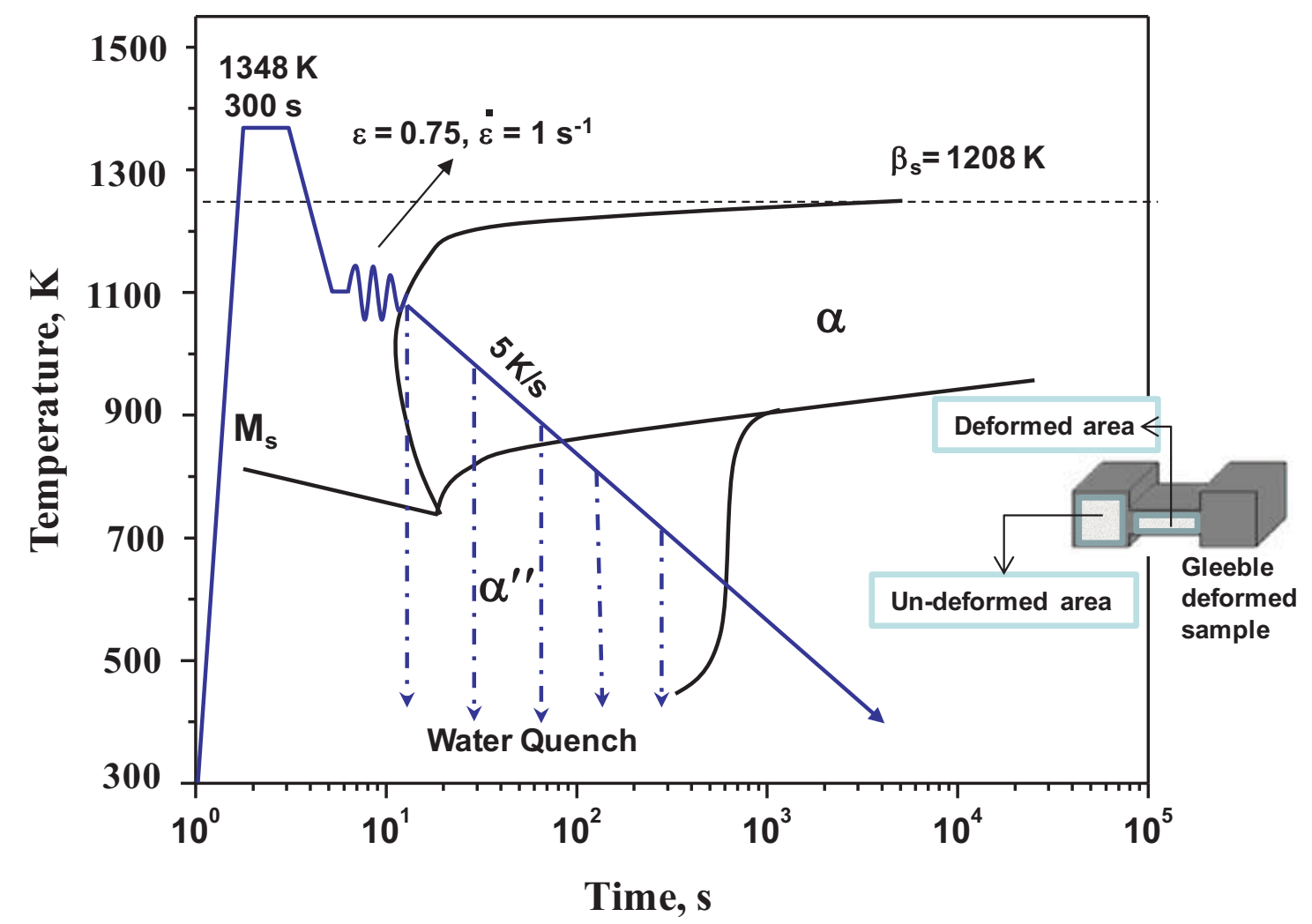

Figure 1 Thermo-mechanical schedules superimposed on a schematic TTT diagram of the Ti-6246 alloy

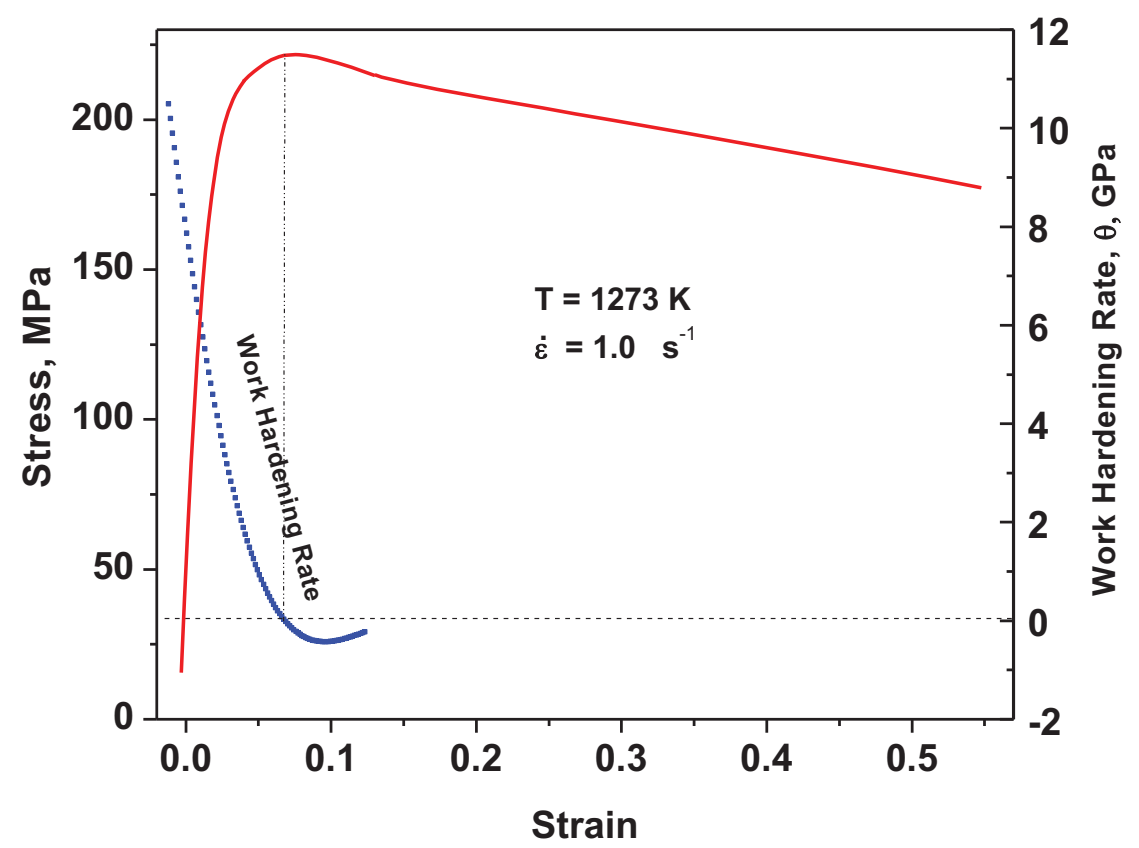


Figure 2 Typical stress-strain curve and work hardening rate of a Ti-6246 alloy following prior deformation at $1073 \mathrm{~K}$ and a strain rate of $1 \mathrm{~s}^{-1}$

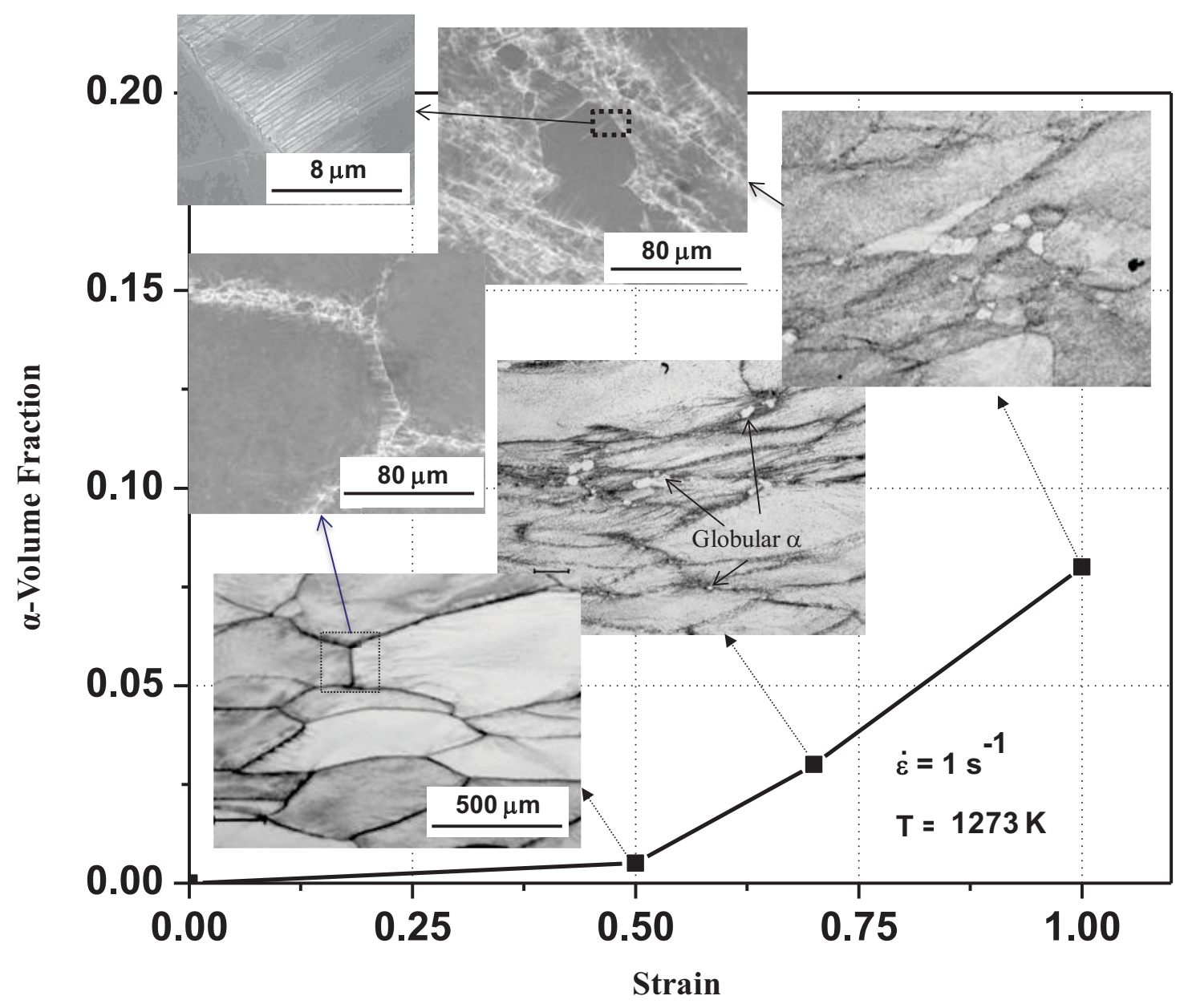

Figure 3 Effect of deformation on the volume fraction and morphology of the $\alpha$-phase 


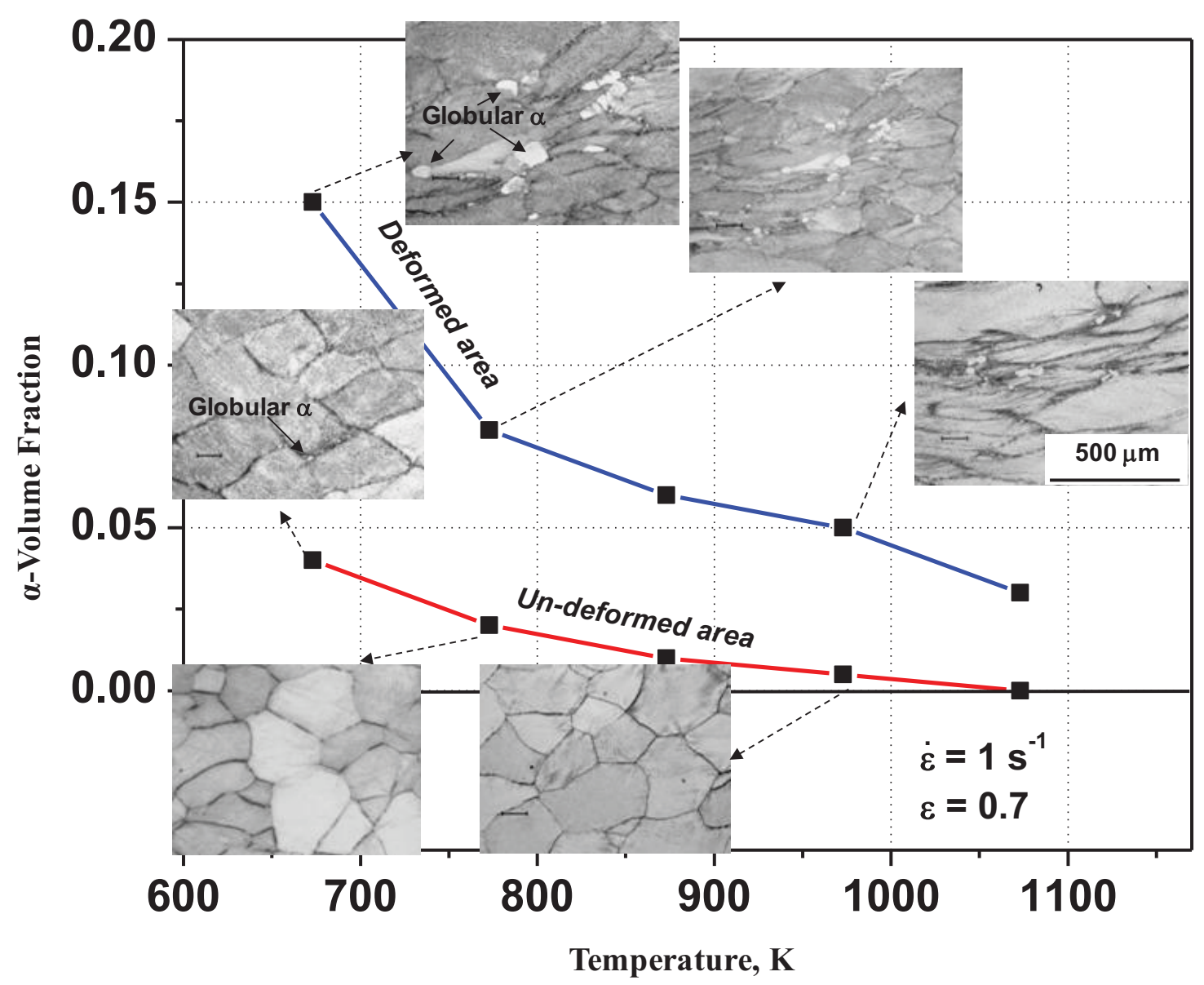

Figure 4 Effect of slow cooling on the volume fraction of $\alpha$ and the morphology of deformed and un-deformed samples
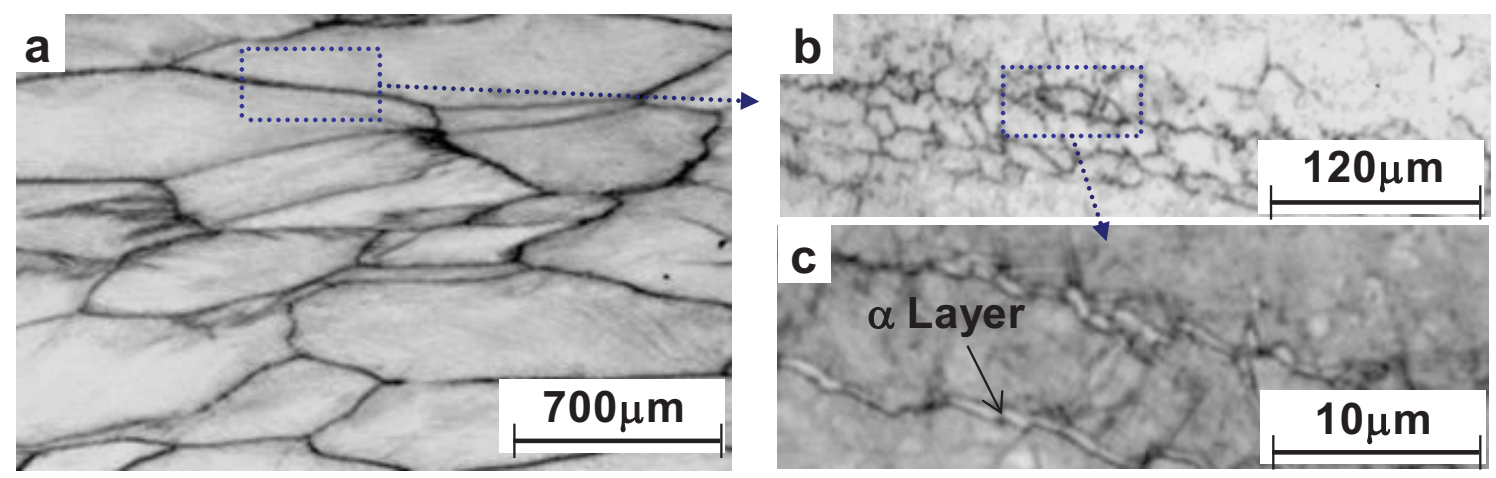

Figure 5 a) Formation of $\alpha$-layers on $\beta$ grain boundaries after deformation at $1075 \mathrm{~K}$, cooling to $873 \mathrm{~K}$ and water quenching. b) DRX on $\beta$-grain boundaries during deformation and c) Formation of $\alpha$-layers on recrystallized $\beta$ - grain boundaries. 


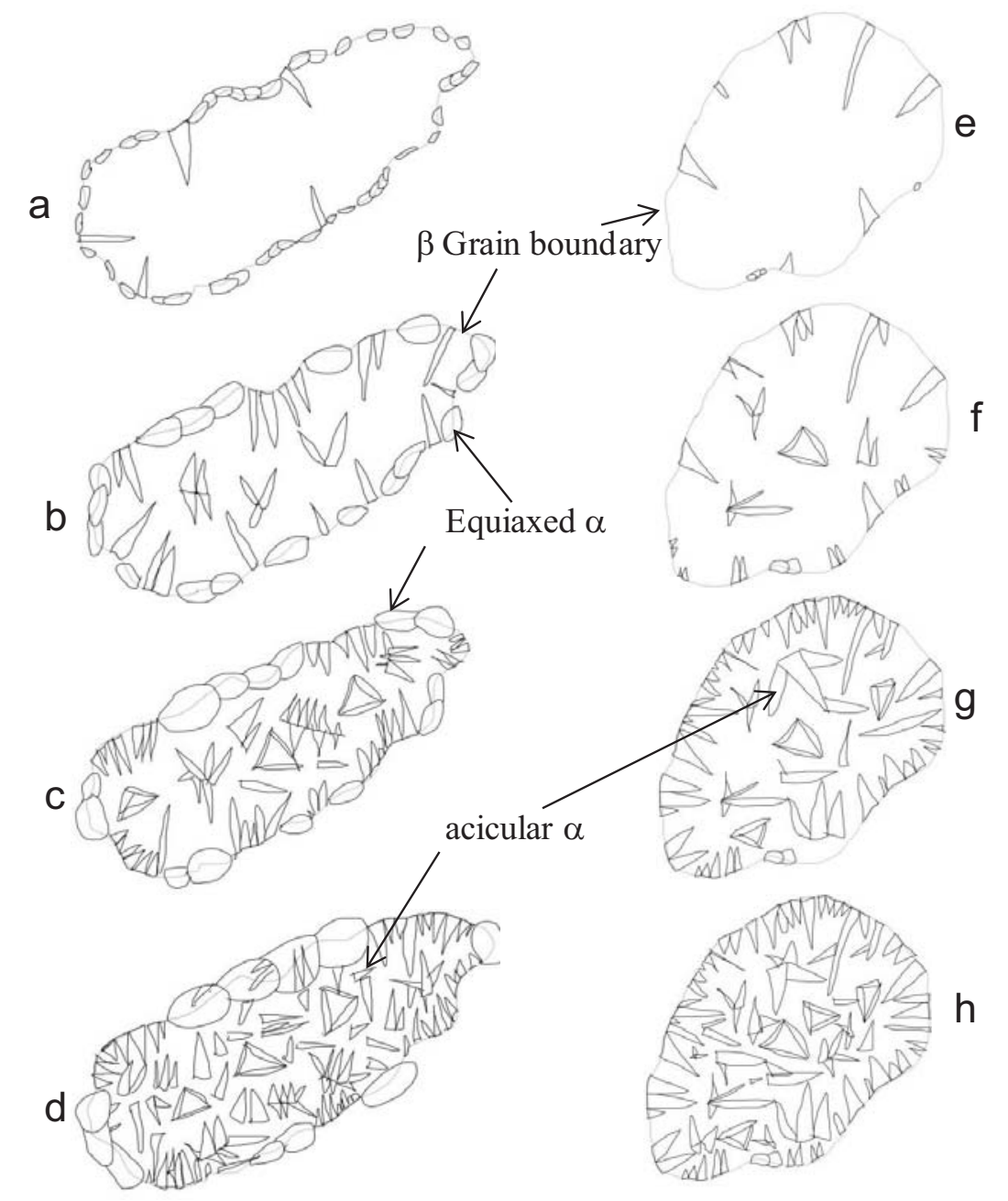

Figure 6 Schematic figure of microstructure development during slow cooling of a deformed (a-d) and un-deformed (e-h) Ti-6246 sample 